\title{
Biomicroscopia ultra-sônica em esclerectomia profunda: relato de caso
}

\author{
Ultrasound biomicroscopy in deep sclerectomy: case report
}

\author{
Flávia Villas-Bôas ${ }^{1}$ \\ Norma Allemann ${ }^{2}$ \\ Maira Saad Avila Morales ${ }^{3}$ \\ Carlos Akira Omi ${ }^{4}$
}

\begin{tabular}{|l|}
\hline RESUMO \\
\hline Relato do caso de um paciente com diagnóstico de glaucoma primário de \\
ângulo aberto, que foi submetida a esclerectomia profunda em olho direito, \\
com sucesso. No pós-operatório de 3 meses, o procedimento cirúrgico foi \\
avaliado com biomicroscopia ultra-sônica (UBM) utilizando-se dois equi- \\
pamentos distintos (UBM840-Zeiss; UBM-VUMAX-Sonomed) com trans- \\
dutores de 50 MHz. O método diagnóstico com biomicroscopia ultra- \\
sônica pode ser usado como método não invasivo para avaliar a arquitetura \\
interna ocular na topografia da esclerectomia profunda. Permite identificar \\
as estruturas do segmento anterior, sua relação anatômica, bem como a \\
membrana trabéculo-Descemet íntegra e o espaço intra-escleral. Biomi- \\
croscopia ultra-sônica demonstrou utilidade na avaliação pós-operatória \\
do procedimento cirúrgico.
\end{tabular}

Descritores: Microscopia/métodos; Ultra-sonografia/métodos; Esclera/cirurgia; Glaucoma de ângulo aberto/cirurgia; Esclerostomia; Período pós-operatorio; Humanos; Feminino; Idoso; Relatos de casos [Tipo de publicação]

\section{INTRODUÇÃO}

A técnica da esclerectomia profunda está sendo utilizada como alternativa para as cirurgias filtrantes convencionais para controle de glaucoma, com a finalidade de aumentar a segurança ${ }^{(1-2)}$. No Brasil, a esclerectomia profunda teve seus primeiros resultados publicados em 2001 por alguns autores $^{(3)}$.

A trabeculectomia é a cirurgia mais freqüentemente realizada para controle do glaucoma. A esclerectomia profunda tem a vantagem de não penetrar na câmara anterior e de não requerer iridectomia. Este procedimento parece preservar mais as estruturas da câmara anterior, suscitando menor grau de inflamação ocular pós-operatória e apresentar menos complicações como atalamia, inflamação, hifema e progressão de catarata ${ }^{(2,4)}$.

A biomicroscopia ultra-sônica (UBM) fornece detalhes da câmara anterior, do corpo ciliar e do tecido córneo-escleral não obtidos por outros métodos em olhos vivos. Os estudos mostram que o UBM é um instrumento valioso para entender a redução da pressão intra-ocular após a cirurgia para glaucoma $^{(5-6)}$.

\section{RELATO DE CASO}

Identificação: A.S.S., sexo feminino, parda, 65 anos de idade, acompanhada no Serviço de Glaucoma há 17 anos, com diagnóstico de glaucoma primário de ângulo aberto. Apresentou indicação para cirurgia antiglauco- 
matosa em OD, devido a controle insatisfatório da pressão intra-ocular, mesmo em uso de medicações.

Optou-se pela realização de cirurgia de esclerectomia profunda em OD, que sucedeu sem intercorrências. Importante ressaltar que a gonioscopia prévia pôde identificar todas as estruturas até banda do corpo ciliar em ambos os olhos. A técnica cirúrgica consistiu em dissecção conjuntival de base fórnice, seguida da colocação subconjuntival de substância antimitótica. Realizou-se a dissecção do retalho escleral superficial de 5 x $5 \mathrm{~mm}$, com base limbar até atingir $1 \mathrm{~mm}$ em córnea clara. Um segundo retalho escleral mais profundo, foi dissecado e excisado, deixando uma fina camada escleral sobre a coróide e o corpo ciliar. Após abertura do canal de Schlemm, retirou-se uma fina lâmina de tecido trabecular deixando-se uma camada contendo trabeculado e membrana de Descemet, permitindo o porejamento de humor aquoso para o meio externo. Após aproximação do retalho escleral realizou-se a sutura da conjuntiva para evitar vazamento do líquido drenado ${ }^{(3)}$.

A pressão intra-ocular manteve-se controlada durante todo o acompanhamento em cerca de $9 \mathrm{mmHg}$, inclusive na medida mais recente, $3^{\circ}$ mês pós-operatório $(10 \mathrm{mmHg})$. À biomicroscopia, a bolha filtrante apresentava aspecto difuso e discretamente elevado.

No $3^{\circ}$ mês pós-operatório OD foi submetido à biomicroscopia ultra-sônica (ultra-sonografia de alta freqüência, transdutores de $50 \mathrm{MHz}$, técnica de imersão). O exame permitiu observar a disposição das seguintes estruturas: "flap" ou retalho escleral superficial, espaço intra-escleral, bolha filtrante elevada, membrana trabéculo-Descemet superiormente (Figuras 1 e 2).

Para o exame, foram utilizados dois equipamentos de ultrasonografia de $50 \mathrm{MHz}$ (UBM 840 - Zeiss Humphrey; UBM Vumax - Sonomed). O exame foi precedido pela instilação de anestésico tópico, com a adaptação de uma cuba de polimetilmetacrilato entre as pálpebras, preenchida com metilcelulose para o UBM Zeiss Humphrey. Para o Vumax Sonomed, foi utilizada uma cuba de silicone preenchida com solução salina a $0,9 \%$. Ambos equipamentos utilizaram o transdutor de alta freqüência de $50 \mathrm{MHz}$. O campo da imagem promovido pelo equipamento UBM 840 - Zeiss Humphrey é de 5,0 (excursão lateral do transdutor) x 5,0 $\mathrm{mm}$ (profundidade). O equipamento UBM Vumax - Sonomed permite a imagem com extensão de $13 \mathrm{~mm}$ e profundidade de $14 \mathrm{~mm}$. O transdutor foi posicionado perpendicularmente à estrutura estudada em ambos os aparelhos.

\section{DISCUSSÃO}

O principal sítio de resistência ao humor aquoso, em olhos portadores de glaucoma primário de ângulo aberto, está na porção justa-canalicular do trabeculado ${ }^{(7)}$.

A cirurgia não penetrante remove o tecido justa-canalicular, levando à redução da PIO sem penetrar na câmara anterior. A pressão intra-ocular obtida pode ser similar ou mais elevada em relação àquela alcançada com a trabeculectomia convencional $^{(7)}$.

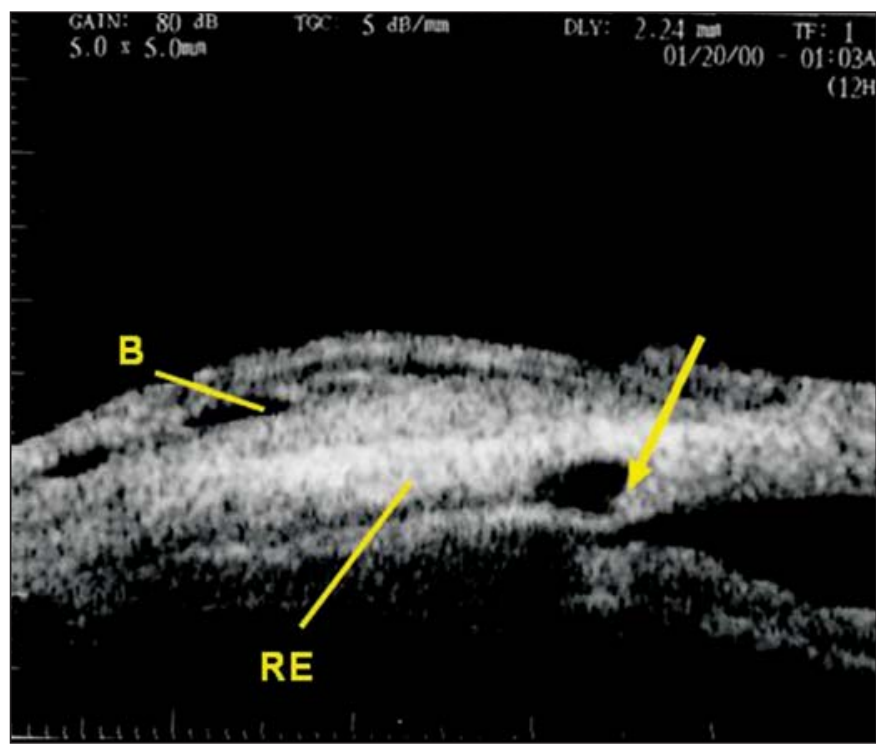

Figura 1 - UBM Zeiss Humphrey. Corte longitudinal demonstra "flap" ou retalho escleral superficial (RE), o espaço intra-escleral, uma bolha filtrante elevada (B), e a membrana trabéculo-Descemet íntegra (seta).

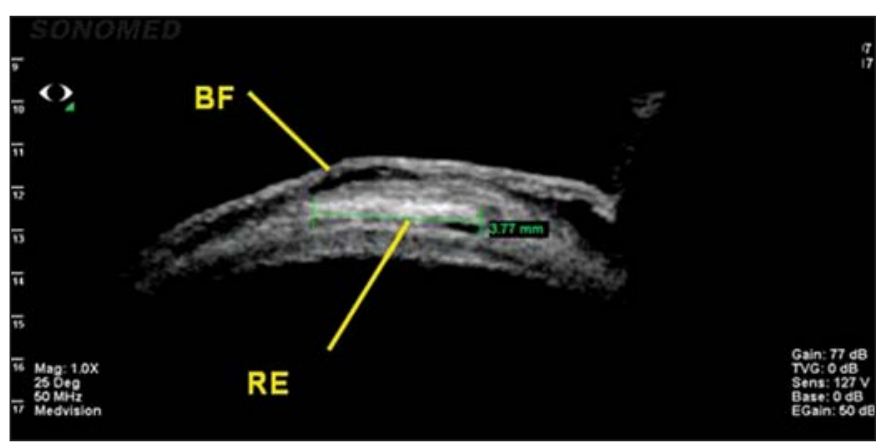

Figura 2 - UBM Vumax. Corte transversal: largura do "flap" escleral superficial medindo $3,77 \mathrm{~mm}$ e bolha filtrante difusa com grande extensão látero-lateral.

A esclerectomia profunda tem como proposta a redução da PIO por inúmeros mecanismos, os quais incluem: criação de uma bolha subconjuntival, remoção de parte do tecido justacanalicular, filtração pelo espaço supraciliar/supracoroidal, dentre outros ${ }^{(8)}$.

O UBM pode ser usado como um método não invasivo para avaliar a arquitetura interna ocular na topografia da esclerectomia profunda após a cirurgia. Permite identificar as estruturas e sua disposição, assim como também permite identificar a presença de uma membrana trabéculo-Descemet íntegra e do espaço intra-escleral, o que demonstra a funcionalidade da $\operatorname{cirurgia}^{(5)}$.

Estudos que compararam a espessura do espaço intraescleral e o nível da pressão intra-ocular não acharam uma correlação estatística significativa. Também não foi observada correlação entre o nível de PIO e a espessura da membrana trabéculo-Descemet remanescente ${ }^{(9)}$. 
As bolhas subconjuntivais filtrantes após a esclerectomia profunda são de refletividade variável, predominantemente baixa. Entretanto, a ausência de bolha filtrante ou a presença de pequena bolha plana não parecem estar relacionadas com a falta de funcionalidade da cirurgia ${ }^{(6,8)}$.

Alguns autores detectaram bolhas filtrantes em $90 \%$ ( $n=22$ olhos) dos casos submetidos a esclerectomia profunda. Os pacientes foram submetidos à biomicroscopia ultra-sônica após $12 \pm 5,3$ meses da cirurgia com o aparelho UBM 840 Zeiss Humphrey. As bolhas foram classificadas em: alta refletividade, baixa refletividade, encapsulada e plana. As bolhas de alta refletividade foram as mais comuns (50\%). A presença e o tipo de bolha não se relacionaram com o sucesso cirúrgico. Muitos casos com bolhas encapsuladas ou planas apresentaram PIO baixa. A reprodutibilidade interobservador também foi analisada no estudo através de múltiplas medidas no mesmo aparelho e foi considerada alta ${ }^{(9)}$.

O caso relatado mostrou os aspectos morfológicos de uma cirurgia de esclerectomia não penetrante a partir de imagens de UBM realizadas por dois tipos de aparelhos atualmente disponíveis. Entretanto, estudos para avaliar os resultados dessa técnica cirúrgica a longo prazo devem ser conduzidos, na tentativa de melhor correlacionar os resultados anatômicos e funcionais, ressaltando a importância do UBM para a melhor compreensão dos mesmos.

Estudos recentes ressaltam a aplicabilidade da tomografia de coerência óptica na avaliação de bolhas filtrantes de trabeculectomia e esclerectomia profunda ${ }^{(10)}$.

Este método diagnóstico foi recentemente introduzido no mercado oftalmológico e apresenta a vantagem de não exigir meio de imersão, ao mesmo tempo em que o paciente pode ter seu olho estudado em posição sentada. No futuro a comparação de ambos métodos permitirá estabelecer padrões de funcionalidade para cada técnica cirúrgica anti-glaucomatosa.

\section{ABSTRACT}

Case report of a woman with the diagnosis of primary openangle glaucoma who was submitted to a successful surgical procedure of deep sclerectomy in the right eye. In the posto- perative period, at month 3 , the surgical procedure was evaluated with ultrasound biomicroscopy (UBM) utilizing two distinct equipments (UBM 840-Zeiss; UBM-VUMAX-Sonomed), with $50-\mathrm{MHz}$ transducers. The diagnostic method of ultrasound biomicroscopy can be utilized as a non invasive method to evaluate the internal architecture of the eye at the topography of deep sclerectomy. It allows to identify the anterior segment structures, their anatomical relationship, and also the intact trabecular-Descemet membrane and the intrascleral space. Ultrasound biomicroscopy showed to be useful in evaluating postoperative status of the surgical procedure.

Keywords: Microscopy/methods; Ultrasonography/methods; Sclera/surgery; Glaucoma, open-angle/surgery; Sclerostomy; Postoperative period; Humans; Female; Aged; Case reports [Publication type]

\section{REFERÊNCIAS}

1. Roy S, Mermoud A. [Complications of deep nonpenetrating sclerectomy]. J Fr Ophtalmol. 2006;29(10):1180-97. French.

2. Roy S, Mermoud A. [Deep nonpenetrating sclerectomy: technical aspects.] J Fr Ophtalmol. 2006;29(10):1160-6. French.

3. Guedes VMP, Guedes RAP. Esclerectomia profunda não perfurante: resultados em médio prazo dos primeiros pacientes operados. Rev Bras Oftalmol. 2001; 60(1):20-4.

4. El Sayyad F, Helal M, El-Kholify H, Khalil M, El-Maghraby A. Nonpenetrating deep sclerectomy versus trabeculectomy in bilateral primary open-angle glaucoma. Ophthalmology. 2000;107(9):1671-4. Comment in: Ophthalmology. 2001;108(10):1718.

5. Marchini G, Marrafa M, Bruneli C, Morbio R, Bonomi L. Ultrasound biomicroscopy and intraocular-pressure lowering mechanisms of deep sclerectomy with reticulated hyaluronic acid implant. J Cataract Refract Surg. 2001;27(4): 507-17.

6. Chiou AG, Mermoud A, Underdahl JP, Schnyder CC. An ultrasound biomicroscopic study of eyes after deep sclerectomy with collagen implant. Ophthalmology. 1998;105(4):746-50.

7. Mermoud A, Vaudax J. Aqueous humor dynamics in non-penetrating filtering surgery. Invest Ophthalmol Vis Sci; 1997:38(Suppl):S1064.

8. Roters S, Luke C, Jonescu-Cuypers CP, Engels BF, Jacobi PC, Konen W, et al. Ultrasound biomicroscopy and its value in predicting the long term outcome of viscocanalostomy. Br J Ophthalmol. 2002;86(9):997-1001.

9. Khairy HA, Atta HR, Green FD, van der Hoek J, Azuara-Blanco A. Ultrasound biomicroscopy in deep sclerectomy. Eye. 2005;19(5):555-60.

10. Labbe A, Hamard P, Iordanidou V, Dupont-Monod S, Baudouin C. [Utility of the Visante OCT in the follow-up of glaucoma surgery]. J Fr Ophtalmol. 2007;30(3):225-31. French. 